\title{
Organisation of the human PAX4 gene and its exclusion as a candidate for the Wolcott-Rallison syndrome
}

\author{
D T Bonthron, N Dunlop, D G D Barr, A A El Sanousi, L I Al-Gazali
}

\begin{abstract}
Neonatal diabetes mellitus is a rare condition, the causes of which are mostly unknown. One well defined though very rare entity is the autosomal recessive Wolcott-Rallison syndrome, in which permanent neonatal diabetes, osteopenia, and epiphyseal dysplasia occur. Only five previous families have been reported, and here we describe the second in which parental consanguinity was present. The proband was born to first cousin parents and died at 2 years from the sequelae of poorly controlled diabetes. To test the hypothesis that mutation of PAX4, required in the mouse for pancreatic islet $\beta$ cell development, might cause WRS, the structure of the human PAX4 gene was deduced and DNA from two unrelated WRS patients sequenced. No PAX4 mutation was present, though the entire coding region was sequenced in both patients. It therefore appears unlikely that PAX4 is involved in the aetiology of WolcottRallison syndrome, though it remains a good candidate for other forms of neonatal diabetes mellitus.

(F Med Genet 1998;35:288-292)
\end{abstract}

Keywords: Wolcott-Rallison syndrome; neonatal diabetes; PAX4; skeletal dysplasia

The combination of permanent neonatal diabetes mellitus and a skeletal dysplasia, manifested early on by osteopenia and fractures, and later by the development of spondyloepiphyseal dysplasia, was first reported in 1972 by Wolcott and Rallison. ${ }^{1}$ Only four further families have been described, three with sib recurrences and one isolated case with a mosaic del(15)(q11-12). ${ }^{2-5}$ Patients have been of both sexes, with normal parents, so that Wolcott-Rallison syndrome (WRS) appears to be an autosomal recessive trait. The clinical outcome in several of the reported cases has been poor, owing either to late diagnosis or poor control of the diabetes mellitus, which seems to be brittle, with extreme insulin sensitivity.

The family of the new patient we describe here is the second from the Arabian peninsula, and also the second in which there has been parental consanguinity. In one previous case of WRS, pancreatic immunohistochemistry showed depletion of insulin staining cells, with the majority of the cells in the prominent islets staining for glucagon. ${ }^{5}$ This is very similar to the picture in the homozygous Pax4 knockout mouse, which dies of lethal $\beta$ cell aplasia. ${ }^{6}$ To test the hypothesis that mutations of human PAX4 might cause WRS, we deduced the exon-intron structure of this gene by comparison to the murine cDNA sequence, and analysed DNA from the new family described here, and of the previously described consanguineous case. ${ }^{4}$ The results indicate that PAX4 is unlikely to be the WRS gene, though it remains a good candidate gene for involvement in other cases of neonatal diabetes, for which the present analysis of the PAX4 intron-exon structure will be useful.

\section{Methods}

ANALYSIS OF PAX4

By BLAST searching with a single published putative human PAX4 exon, ${ }^{7}$ a cosmid (g1572c264) was identified from within the Olson Laboratory chromosome 7q31.3 contiguous sequencing project (http:// chimera.biotech.washington.edu/uwgc/projects/ chr-7/chr7proj.htm). Its $46.6 \mathrm{~kb}$ sequence was then compared to published partial murine Pax4 cDNA $\left(\mathrm{Y}_{\left.09584^{6}\right) \text { and genomic }}^{8}\right.$ sequences using UWGCG BESTFIT. ${ }^{9}$ Together with the results of splice junction prediction using NetGene, ${ }^{10}$ six human PAX4 exons (B-G), encompassing the entire paired and homeo domains, could be unambiguously identified. A similar analysis using additional unpublished murine cDNA sequence ( $L$ St Onge, $P$ Gruss, unpublished data) allowed identification of four further exons. Based on this exonintron structure, six primer pairs ( $0 \mathrm{f} / \mathrm{r}$ to $5 \mathrm{f} / \mathrm{r}$ ) were used to amplify the gene in segments (fig 1) and PCR products were sequenced directly using the remaining primers and $\alpha^{33} \mathrm{P}$-ddNTP or dye terminator Thermosequenase kits (Amersham).

The PAX4 primer sequences were: of: CAGCTGGAGACAACAGCTGTCCAT, 0r: GTTGATGGAAGCAAAGCCCTGAGG; 1f: CCGTGAGCCAGCTCTCAAAGAAAGCAGCTT, 1r: AAACTTATGGGTCAGAATTGCCCAGGAGCC; 2f: AAGGGCAACATCCTCAGGGTATAGCCCTGA，2r: TG СТTCСAAT TTCTCACСТCСССТСТССAC; 3f: GATGTCAGGCCCAAGGAAGG GTCAA, 3r: GATGACTGAGCGGGCAGATGGATG; 4f: TTCACACCCCTGCAAGAAGTGGCT, 4r: AATGGAGATCCATGCCCTCCTGGA; 5f: CTGCCCACTACTGTATGGCTTGGA, 5r: AGAGTGGGCATAGGGGTGCTCATA; 1s1: GCTCTTGCCTTCAGAGGA, 1s2: CCCAGGGACACTCTCCTT; 


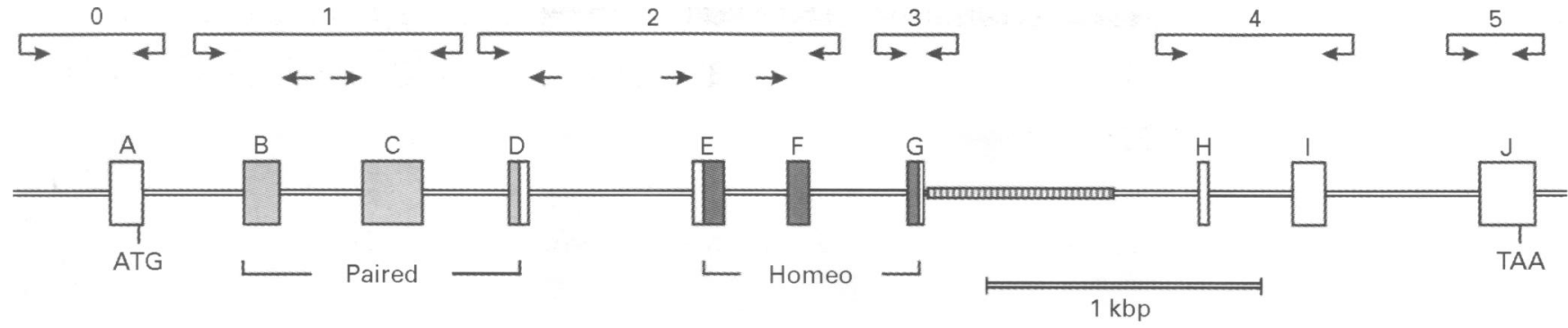

Hu: TPPVVARIAQLKGECPALFAWEIQRQLCAEGLCTQDKTPSVSSINRVLRALQEDQGLPCTRLRSPAVLAPAVLTPHSGSE

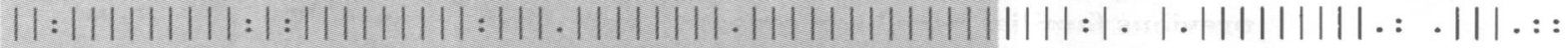

Mu: TPAVVARIAQLKDEYPALFAWEIOHQLCTEGLCTODKAPSVSSINRVLRALQEDQSLHWTQLRSPAVLAPVLPSPHSNCG

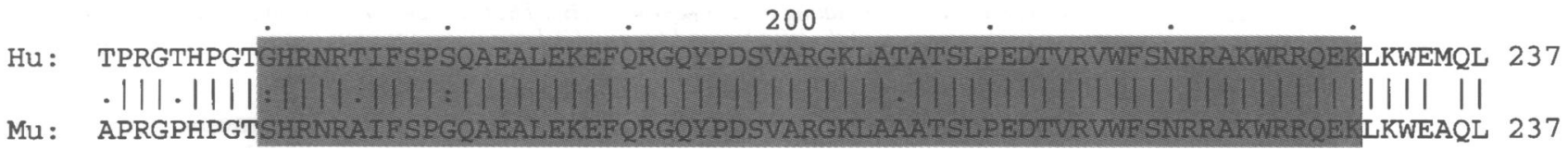

Figure 1 Partial structure of the human PAX4 gene, deduced from the sequence of cosmid g1572c264. Exons and introns are to scale; the exons are provisionally labelled $A-\mathcal{F}$, since the full extent of the gene (in particular of any additional 5 ' exons, as in PAX6) is not known for certain. The predicted exon positions are at $n t$ [31545]-31434, 31049-30919, 30613-30398, 30075-30000, 29399-29274, 29049-28967, 28608-28539, 27525-27470, 27201-27060, and 26510-26326 of the cosmid. The 5' boundary of the first of these exons $(A)$ cannot be defined with certainty, as indicated by the parentheses. The paired and homeo domains are shaded respectively light and dark grey, both in the diagram and in the sequence alignment, in which predicted amino acid sequences of human (Hu:) and mouse (Mu:) PAX4 are compared. The hatched box downstream of exon G indicates a tetranucleotide repeat sequence. Oligonucleotide primers (see Methods) are indicated by arrows, with the pairs used for PCR joined by brackets.

2s1: ACGAGAAAGGGCTTTGAG, 2s2: AGCCCCAGAGACTCAGGGA; 2s3: TTGGGGTTGTAGCAGGTGG.

\section{Case report}

The proband was born at 28 weeks' gestation, weighing $1100 \mathrm{~g}$, the fourth child of first cousin Saudi parents. The previous three sibs were healthy. Proptosis, a wide anterior fontanelle, and high arched palate were noted at birth, and the baby was ventilated for respiratory distress. On day 4, a blood glucose of 22.3 $\mathrm{mmol} / \mathrm{l}$ and glycosuria were noted and insulin treatment begun. Before this, blood glucose had been normal. The insulin was discontinued on day 28 and extubation achieved on day 35.

Bilateral femoral fractures occurred on day 72 , at which time the baby's sclerae were noted to be blue, and a presumptive diagnosis of osteogenesis imperfecta was made. He was discharged home apparently well on day 90 , but readmitted at 4 months with severe dehydration owing to diabetic ketoacidosis and a blood glucose of $50.8 \mathrm{mmol} / \mathrm{l}$. Subsequent diabetic control was poor, with frequent hypo- or hyperglycaemia, and when seen at 8 months there was gross motor delay and a spastic diplegia with bilateral ankle clonus.

At 11 months the patient weighed $5 \mathrm{~kg}$. $\mathrm{He}$ was hypotonic and immobile in the supine position. There was marked anteroposterior head flattening, with wide open sutures and fontanelles. There was proptosis, and the palpebral fissures slanted downwards mark- edly. The sclerae were grey/blue, the palate high arched, and teeth absent. The chest was also flattened anteroposteriorly and there was a thoracolumbar kyphosis. Both femora were bowed (fig 2B). A CT scan showed gross ventricular dilatation.

Review of $x$ rays performed at this time showed brachycephaly with poor ossification of the skull and Wormian bones. Long bones were undertubulated, with thin cortices and osteopenia (fig 2B). There was a thoracolumbar kyphosis (fig 2C) and platyspondyly.

During his second year, the proband was admitted several times on account of respiratory infections. His diabetes was managed with twice daily isophane insulin. There was severe psychomotor delay. He was referred elsewhere for neurosurgical assessment of his hydrocephalus, but before that developed urinary tract infection with severe diabetic ketoacidosis and died aged 2 years.

A further healthy sib was born after the index case. The sixth child in the family, now aged 5 months, was then born at 30 weeks' gestation, weighing $1500 \mathrm{~g}$. He presented at 25 days with diabetic ketoacidosis, and has also been diagnosed to have permanent diabetes mellitus, currently well controlled on isophane insulin (2 U bid). A skeletal survey at 1 month of age showed normal bones. At 5 months, he weighed $5.5 \mathrm{~kg}$. Psychomotor development is normal. There are no dysmorphic features and sclerae are not blue. The OFC is appropriate for age, and the anterior fontanelle is of normal size. 

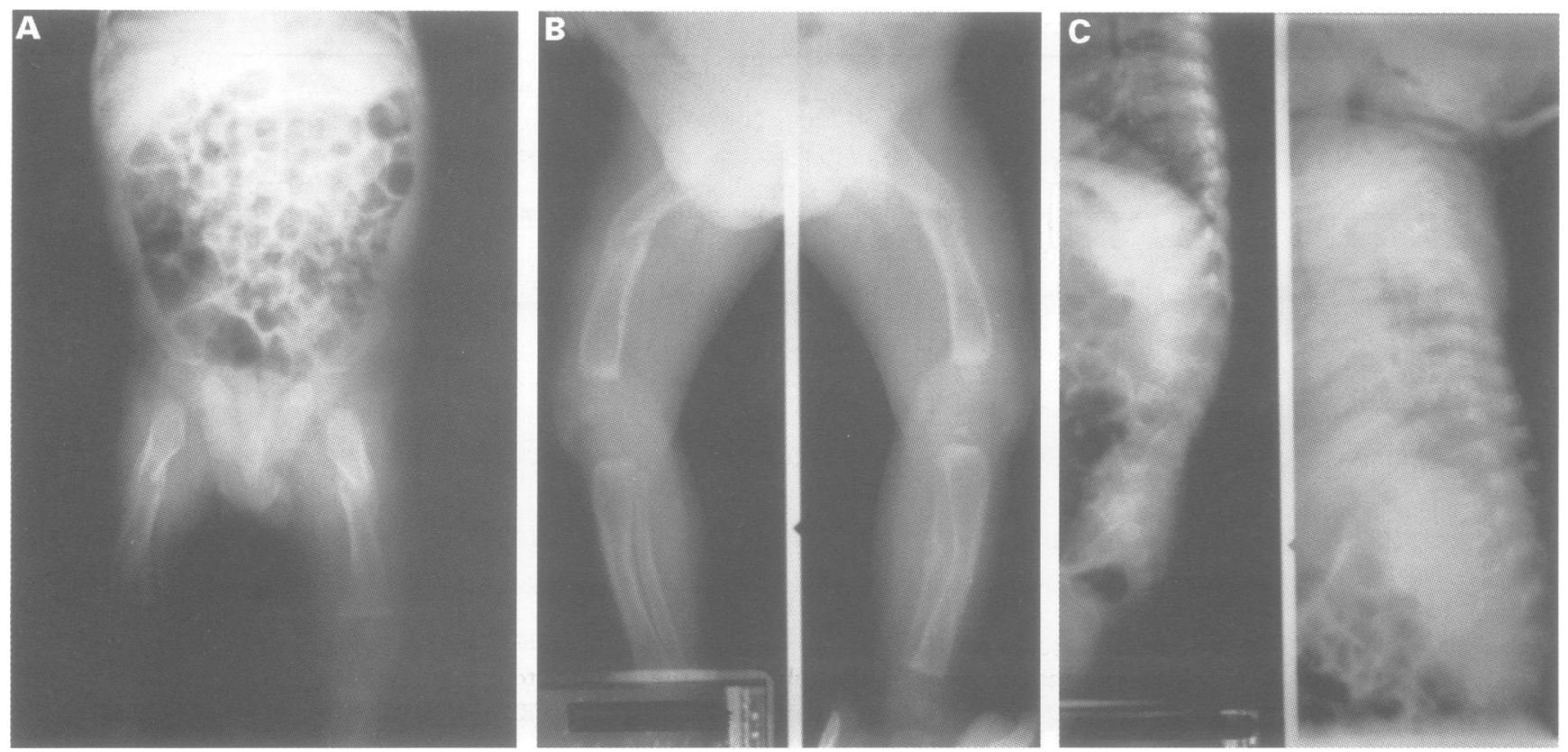

Figure 2 Radiographs of proband. (A) Bilateral femoral fractures, resulting in later deformity (B). The long bones are also abnormally modelled, and show osteopenia with thin cortices. In (B), there is a further fracture of the left tibia and fibula. (C) Lateral spine to show kyphosis in the lumbar region.

\section{Results}

We considered a number of possible aetiologies for WRS. One possibility is homozygosity for a deletion of two or more genes, one involved in pancreatic islet development, and the other in the skeleton. With the osteogenesis imperfectalike features, COL1A1 or COL1A2 could be involved in such a contiguous gene syndrome; homozygous COL1A2 deficiency has been previously seen in severe progressive OI. ${ }^{11}$ However, dermal fibroblast collagen biosynthesis was normal in one previous WRS patient $^{3}$ and the proband was heterozygous for polymorphisms at both COL1A1 and COL1A2, making such a scenario unlikely, given the parental consanguinity. We therefore considered known causes of neonatal diabetes. Paternal uniparental disomy (UPD) of chromosome 6 has been reported in several cases of transient neonatal diabetes mellitus, ${ }^{12}$ as well as in one child who died aged 16 days with agenesis of pancreatic $\beta$ cells. ${ }^{13}$ UPD6 could not account for WRS, though, given the sib recurrences.

Because neonatal diabetes is so rare, it is likely that identification of other genetic defects in this condition may come from experimental studies in the mouse. For example, mutation of the homeodomain transcription factor gene IPF 1 causes pancreatic agenesis in both mouse and human. ${ }^{14}{ }^{15}$ In the present and previous cases of WRS, there was no evidence for an exocrine pancreatic defect, and in one case the pancreas was macroscopically normal, ${ }^{2}$ making IPF1 an unlikely candidate gene. However, it was recently shown that homozygous null mutation of the murine Pax4 gene causes selective $\beta$ and $\delta$ cell agenesis with lethal neonatal diabetes, but preservation of the other components of the pancreas. ${ }^{6}$ This selectivity for the pancreatic islet, and the recessive nature of the mutation, make PAX4 a good candidate gene for WRS.
PAX4 GENE STRUCTURE

Pax4 is one of a family of mouse genes containing the 128 amino acid DNA binding "paired" domain. ${ }^{8}$ Like its closest relative (Pax6), it also contains a second DNA binding homeo domain. The conserved developmental functions of human and mouse Pax genes are indicated by the similar phenotypes owing to mutations of, for example, PAX6 (aniridia) and Pax6 (small eye) or PAX3 (Waardenburg syndrome) and Pax3 (splotch). No human PAX4 sequences are available in public databases, and PAX4 cDNAs, either human or mouse, are not identifiable in dbEST. The scarcity of transcripts has in fact aroused suspicions that Pax 4 might be a pseudogene. ${ }^{8} 16$ A single human exon sequence, similar to Pax4 and located on chromosome 7q32, has however been reported. ${ }^{7}$ Within the University of Washington Chromosome 7q31.3 Sequencing Project, we identified a recently completed 47 $\mathrm{kb}$ sequence (g1572c264, AC000359) containing this putative PAX4 exon, and by splice junction prediction and sequence comparison were able to locate a further nine PAX4 exons within the cosmid. As shown in fig 1, this allowed us to deduce a partial PAX4 gene structure, the 10 identified exons including the entire region coding for the predicted PAX4 protein. As in PAX6, the paired and homeo domains are contained in six exons (B-G), whose close homology to published partial murine Pax4 sequence is shown in fig 1. A short ( $-30-40$ residue) proline rich region between the paired and homeo domains is less well conserved than either of the DNA binding domains flanking it.

ANALYSIS OF PAX4 IN PATIENTS

The primers indicated in fig 1 were used to amplify PAX4 from genomic DNA of the proband and the previously reported case 2 of Al-Gazali et al. ${ }^{4}$ All 10 exons were then directly sequenced. These DNA sequences all proved 
completely identical to that of the g1572c264 cosmid with one exception. In the proband, there was a heterozygous $G \rightarrow A$ substitution in exon $\mathrm{J}$ (corresponding to $\mathrm{C} \rightarrow \mathrm{T}$ at $\mathrm{nt} 26378$ of the g1572c264 sequence). This creates a new stop codon (TGG $\rightarrow$ TAG, W349X) close to the end of the predicted PAX4 protein, removing the last two residues (TrpPro). Definitive interpretation of this sequence change must await isolation of human PAX4 cDNAs, since there is no formal proof as yet (other than the similarity to the mouse sequence) that we have correctly identified the last PAX4 exon. However, the putative W349X change was found to be a common polymorphism, with an allele frequency of 0.15 in Scotland.

Although our failure to find pathogenic mutations leaves a formal possibility of mutations in non-coding regions of PAX4, the 10 exons sequenced appear by comparison to mouse cDNA sequences to encode the whole of the human PAX4 protein. The heterozygosity for W349X in the proband, the product of a consanguineous marriage, is additional evidence against the involvement of PAX4 in WRS.

\section{Discussion}

There are only 10 previously reported cases of the combination of permanent neonatal diabetes and skeletal dysplasia, ' in five families. The evidence strongly indicates autosomal recessive inheritance, since patients have been of either sex, all parents have been normal, sib recurrences have occurred in all but one family, and in both the families we have analysed here, the parents were consanguineous. ${ }^{4}$

The clinical features of WRS were recently reviewed. ${ }^{4}$ Despite the rarity of neonatal diabetes, WRS may be underdiagnosed, since unless fractures occur $x$ rays may not be done. The other radiological features of multiple epiphyseal dysplasia (MED) in WRS evolve with age and are not noticeable at the time when the diabetes presents. In the proband, diabetes presented at 4 days old in a premature infant. Previously, the earliest age of detection of diabetes in WRS has been 2 weeks. Clearly, therefore, the diabetes is congenital, which together with its permanence points to a genetic defect of $\beta$ cell development. Particularly interesting in this regard is the most recent report, the only case in which pancreatic histology was performed. ${ }^{5}$ In addition to general hypoplasia of the pancreas, there was prominence of the islets and abundance of glucagon staining $\alpha$ cells, at the expense of $\beta$ cells. This is extremely similar to the histological abnormality seen in the Pax4 -/mouse ${ }^{6}$ and prompted our analysis of PAX4 in two WRS families.

Including the new family described here, two out of six reported WRS families are from the Arabian peninsula. It is possible therefore that an increased frequency of WRS occurs in this ethnic group. The poor outcome in the proband is similar to that of three previous patients, and can be attributed to inadequate diabetic control with frequent swings between hypoglycaemia and ketoacidosis.

Though previous reports have emphasised MED as the major skeletal feature of WRS, an osteogenesis imperfecta-like picture may occur early on, as in this report. The original case 1 of Wolcott and Rallison ${ }^{1}$ was especially similar to our proband, with three femoral fractures in the first year and persistent diffuse demineralisation of the bones; epiphyseal dysplasia and a metaphyseal modelling defect were only noted at 3 years. Case 2 in that family had no fractures reported and no $x$ rays were performed; case 3 was found to have MED at 14 months of age. Goumy et al also emphasised the widespread osteoporosis in WRS. Like the second case in our family, one presumed case in their family, though diabetic, had normal skeletal radiology at 3 weeks of age. Femoral fracture also occurred in one other patient. ${ }^{5}$

A lethal autosomal recessive phenotype, characterised by neonatal diabetes resulting from lack of pancreatic islet $\beta$ (and $\delta$ ) cells, occurs when the mouse Pax4 gene is mutated. ${ }^{6}$ Since to our knowledge WRS is the only proven autosomal recessive human disorder in which neonatal diabetes mellitus occurs, PAX4 was an obvious candidate gene for WRS. We have deduced the PAX4 gene structure from anonymous cosmid sequence. In view of the absence of PAX4 from dbEST, human PAX4 expression seems, like that of mouse Pax4, to be highly restricted. However, the rarity of polymorphic difference between either patient and reference cosmid (over the 2500 bp sequenced), and the close similarity between the predicted human and mouse PAX4 protein sequences, support the conclusion of the mouse knockout experiments, that despite difficulties in identifying transcripts, this gene is essential. The combination of PAX4 heterozygosity in one consanguineous family and the absence of other mutations, however, indicate that PAX4 is not the WRS gene. Further analysis of this gene in non-syndromic cases of neonatal diabetes is indicated.

Work in DTB's laboratory is supported by the MRC and the Wellcome Trust. We thank Beverley Aldridge (University of Oxford) for type I collagen polymorphism typing of the proband. We are very grateful to Luc St Onge and Peter Gruss (Max Planck Institute, Göttingen, Germany) for making available unpublished murine Pax4 cDNA sequence.

1 Wolcott CD, Rallison ML. Infancy-onset diabetes mellitus and multiple epiphyseal dysplasia. F Pediatr 1972;80:292-7. 2 Goumy P Maroteaux P, Stanescu V, Stanescu R, Labbe A Menut G. Syndrome de transmission récessive autosomique, anociant un diabète congénital et des désordres de la croissance des épiphyses. Arch Fr Pediatr 1980;37 323-8.

3 Stöss H Pesech HJ, Pontz B, Otten A, Spranger J. WolcottRallison syndrome: diabetes mellitus and spondyloepiphyseal dysplasia. Eur f Pediatr 1982;138:120-9.

4 Al-Gazali LI, Makia S, Azzam A, Hall CM. WolcottRallison syndrome. Clin Dysmorphol 1995;4:227-33.

5 Stewart FJ, Carson DJ, Thomas PS, Humphreys $M$, Thorn ton $M$, Nevin NC. Wolcott-Rallison syndrome associated with congenital malformations and a mosaic deletion 15q11-12. Clin Genet 1996;49:152-5.

6 Sosa-Pineda B, Chowdhury K, Torres M, Oliver G, Gruss P. The Pax4 gene is essential for differentiation of insulinproducing $\beta$ cells in the mammalian pancreas. Nature 997;386:399-402.

7 Tamura T, Izumikawa $Y$, Kishino T, Soejima $H$, Jinno $Y$, Niikawa N. Assignment of the human PAX4 gene to chromosome band $7 \mathrm{q} 32$ by fluorescence in situ hybridization. Cytogenet Cell Genet 1994;66:132-4.

Wlather C, Guenet JL, Simon D, et al. Pax: a murine family of paired box-containing genes. Genomics 1991;11:424-34. 9 Devereux J, Haeberli P, Smithies O. A comprehensive set of sequence analysis programs for the VAX. Nucleic Acids Res 1984;12:387-95.

10 Brunak S, Engelbrecht, J Knudsen S. Prediction of human mRNA donor and acceptor sites from the DNA sequence. f Mol Biol 1991;220:49-65. 
11 Nicholls AC, Osse G, Schloon HG, et al. The clinical features of homozygous $\alpha 2(\mathrm{I})$ collagen deficient osteogenfeatures of homozygous $\alpha 2(I)$ collagen deficient
esis imperfecta. $\mathcal{F}$ Med Genet 1984;21:257-62.

12 Temple IK, Gardner RJ, Robinson DO, et al. Further evidence for an imprinted gene for neonatal diabetes localised to chromosome 6q22-q23. Hum Mol Genet 1996;5: 1117-21

13 Abramowicz MJ, Andrien M, Dupont E, et al. Isodisomy of chromosome 6 in a newborn with methylmalonic acidemia and agenesis of pancreatic beta cells causing diabetes mellitus. $\mathcal{F}$ Clin Invest 1994;94:418-21.
14 Jonsson J, Carlsson L, Edlund T, Edlund H. Insulinpromoter-factor 1 is required for pancreas development in promoter-factor 1 is required for pancreas development in
mice. Nature 1994;371:606-9.

mice. Nature 1994;371:606-9.
15 Stoffers DA, Zinkin NT, Stanojevic V, Clarke WL, Habener JF. Pancreatic agenesis attributable to a single nucleotide deletion in the human IPF1 gene coding sequence. Nat

16 Sun $\mathrm{H}$, Rodin A, Zhou Y, et al. Evolution of paired domains: isolation and sequencing of jellyfish and hydra Pax genes related to Pax-5 and Pax-6. Proc Natl Acad Sci USA 1997; 94:5156-61. 